\title{
Green Computing and Communications for Smart Portable Devices
}

\author{
Jun Huang $\mathbb{D}^{1}{ }^{1}$ Zhi Liu, ${ }^{2}$ Qiang Duan, ${ }^{3}$ Mohammed Atiquzzaman, ${ }^{4}$ \\ Minho Jo $\left(D,{ }^{5}\right.$ and Zygmunt J. Haas ${ }^{6}$ \\ ${ }^{1}$ Chongqing University of Posts and Telecommunications, Chongqing, China \\ ${ }^{2}$ Waseda University, Tokyo, Japan \\ ${ }^{3}$ Penn State University, Abington College, Abington, PA, USA \\ ${ }^{4}$ University of Oklahoma, Norman, OK, USA \\ ${ }^{5}$ Korea University, Seoul, Republic of Korea \\ ${ }^{6}$ University of Texas at Dallas, Richardson, TX, USA
}

Correspondence should be addressed to Jun Huang; xiaoniuadmin@gmail.com

Received 6 March 2018; Accepted 6 March 2018; Published 15 April 2018

Copyright (c) 2018 Jun Huang et al. This is an open access article distributed under the Creative Commons Attribution License, which permits unrestricted use, distribution, and reproduction in any medium, provided the original work is properly cited.

Smart portable devices have been playing a crucial role in the long-term evolutionary (LTE) communications and are expected to be an indispensable part of the 5G system. While smart portable devices (e.g., smartphone and tablets) have powerful computing and communication capabilities, the high data rate services, however, drain out the energy of the device much faster than before. For example, a mobile user can watch a video on a smartphone, to provide an ad for an interesting item, and tap through the site to purchase it on the phone. However, the current phone battery is not durable to support all these activities in one day. Even worse, if a user typically transmits/shares large volumes of data for a long time, it will shorten the lifetime of the device significantly and deplete the batteries rapidly. As a result, cost-effective and energy-efficient computing and communication mechanism for smart portable devices are required to be designed and developed.

With the goal of reduction of energy consumption, green computing and communication refer to the solutions that are able to improve the network's performances in terms of computing/energy efficiency, data privacy, and network security. This special issue intends to bring together the leading researchers and developers from both academia and industry to discuss and present their views on all the aspects of green computing and communications for smart portable devices.
This special issue attracted twenty submissions, and eight papers are accepted to be published after the peer-review process with at least three reviewers base on originality, quality, and relevance to this special issue and the journal. The first paper "Greening Software Requirements Change Management Strategy Based on Nash Equilibrium" by Z. Tong et al. looked into the green computing, in software engineering, and proposed effective strategies to manage the requirement change based on game theory.

The second paper "Energy Efficient Downlink Transmission in Wireless LANs by Using Low-Power Wake-Up Radio" by S. Tang and S. Obana studied the transmission scheduling in the downlink of a WLAN by using a wake-up radio to remotely activate a WLAN module to receive packets on demand. The carefully designed scheme reduces the power consumption and results in less delay and higher throughput.

The third paper "Multihop Data Delivery Virtualization for Green Decentralized IoT" by L. Zhang et al. studied green decentralized communication technologies for IoT and proposed a multihop data delivery virtualization approach which uses multiple one-hop reliable transmissions to perform multihop data transmissions. The real world experiments were conducted besides the computer simulations and higher throughput and shortened transmission time can be observed. 
The fourth paper "A Game Theory Based Approach for Power Efficient Vehicular Ad Hoc Networks" by K. Hua et al. investigated the throughput and transmission delay performances for real-time and delay sensitive services through a repeated game theoretic solution towards a power efficient vehicular ad hoc networks.

The fifth paper "Energy Efficiency Analysis of ICN Assisted 5G IoT System" by Di Zhang et al. analyzed the energy efficiency for ICN assisted 5G IoT system with innetwork core router as well as short and long distance scenarios based on the introduced comprehensive system model.

The sixth paper "Energy and Delay Optimization of Heterogeneous Multicore Wireless Multimedia Sensor Nodes by Adaptive Genetic-Simulated Annealing Algorithm" by $X$. Liu et al. researched an energy-efficient, delay-efficient, hardware, and software optimization platform to minimize the energy cost, while guaranteeing the deadline of the realtime WMSN tasks.

The seventh paper "Short-Range Cooperation of Mobile Devices for Energy-Efficient Vertical Handovers" by X. Foukas et al. introduced the concept of short-range cooperation for energy-efficient vertical handovers and developed a model for capturing the associated mean energy expenditure per handover by means of closed-form expressions.

The eighth paper "Green Data Gathering under Delay Differentiated Services Constraint for Internet of Things" by M. Huang et al. studied the energy-efficient data gathering techniques and proposed a delay differentiated services based data routing (DSDR) scheme to improve the delay differentiated services constraint that is missed from previous data gathering studies.

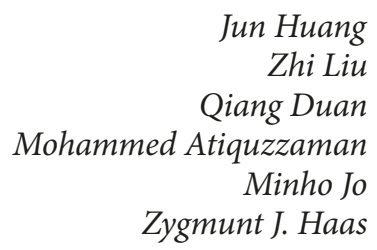




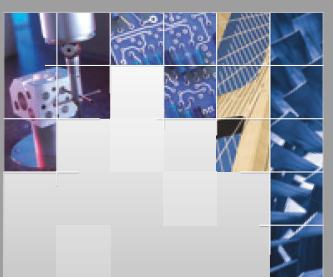

\section{Enfincering}
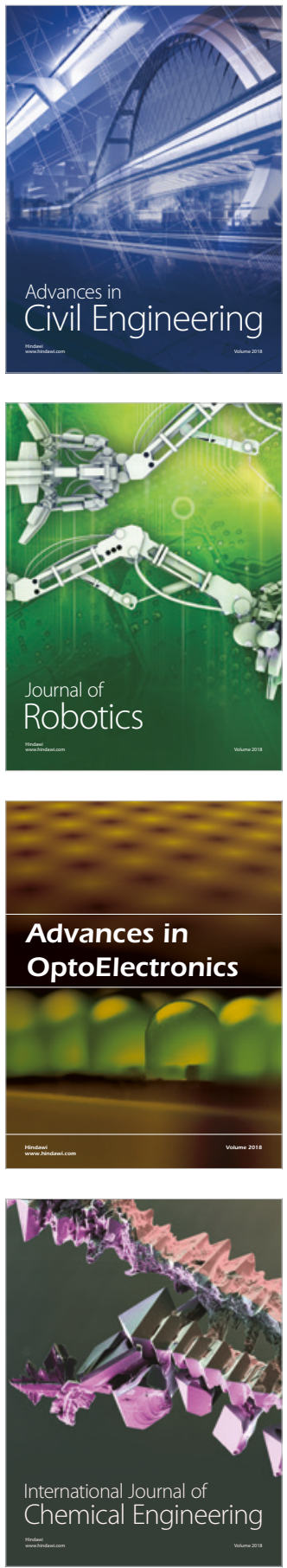

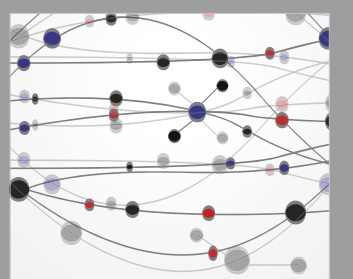

\section{Rotating \\ Machinery}

The Scientific World Journal

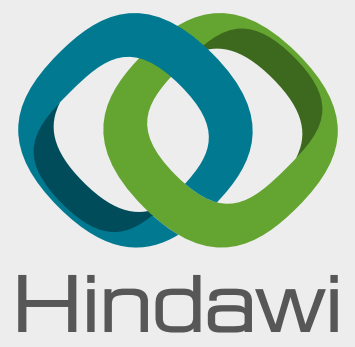

Submit your manuscripts at

www.hindawi.com
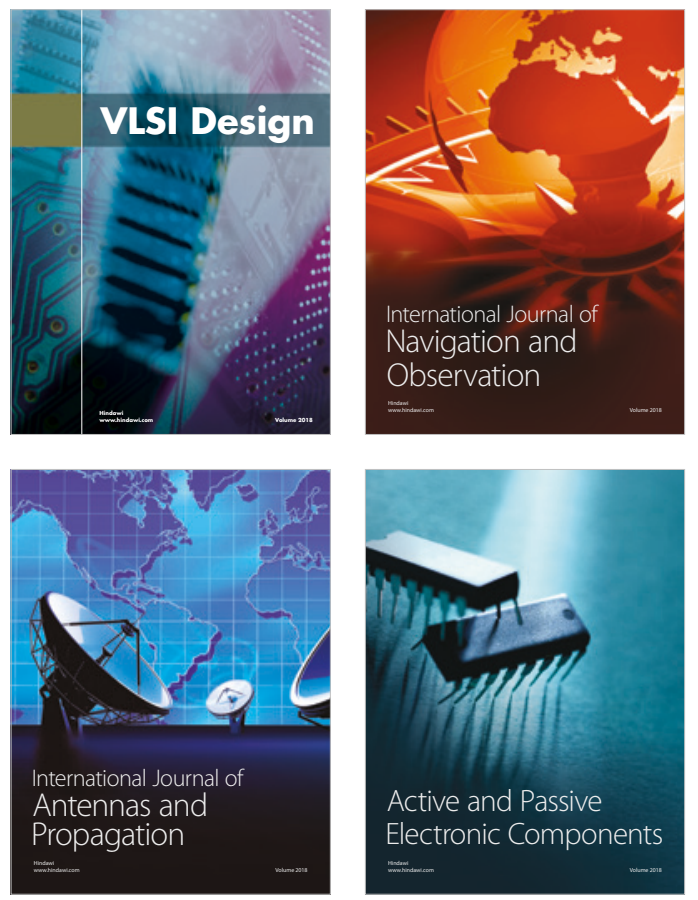
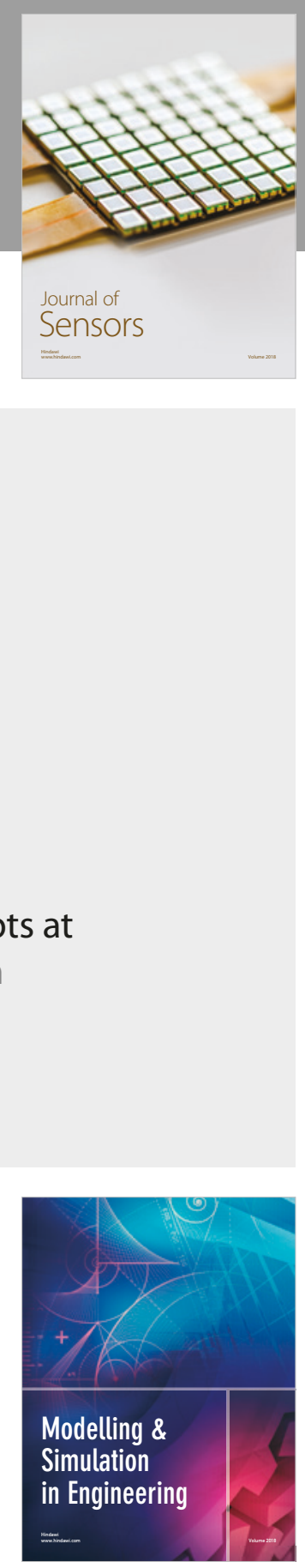

\section{Advances \\ Multimedia}
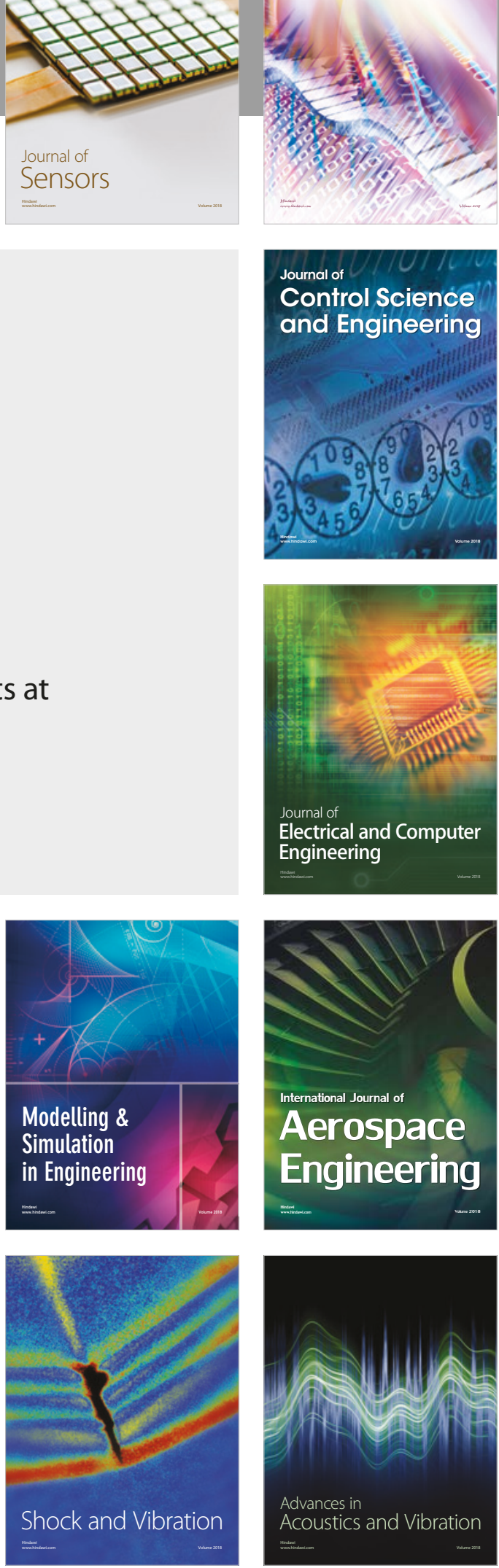